\title{
A NEW SINGLE DISK SPUTTERING SYSTEM FOR MANUFACTURING OF MO-MEDIA (Invited)
}

\author{
G. BRAEUER, B. CORD, W. DICKEN, H. FRANKENBERGER and S. SCHULZ
}

Leybold AG, Wilhelm-Rohn-Strasse 25, 63450 Hanau, Germany

\begin{abstract}
A new single-disk sputtering system for the production of magneto-optical disks is presented. It features 7 process stations for multi-step static deposition which are arranged vertically on a circular path, and which can be equipped with cathodes or an etching station according to the specific needs. Special emphasis is put on the design of the cathodes being completely axialsymmetric in geometry and the sputter gas management. Results from DC-reactive sputtering for fast deposition of dielectric layers are presented with layer uniformities of $\pm 2.0 \%$.

KEYWORDS: MO-DISKS, REACTIVE SPUTTERING, PROCESSING TECHNIQUES
\end{abstract}

\section{INTRODUCTION}

Manufacturing of magneto-optical disks has now surpassed the border of real mass production while, at the same time, the demand for MO disks is growing rapidly. This development is accompanied by a steady increase of storage densities and cost pressures on production. Equipment dedicated to the manufacturing of media must therefore cover the specific aspects of this situation.

The requirements for MO-disk production can be divided into two parts, the technological (including appropriate layer properties) and economical ones.

The technological aspects cover:

- films with strict tolerances of material properties

- constant films properties (including thickness) over the disks recording area

- low particle and defect generation

- high stability and reproducibility.

The economical factors are:

- high degree of automatization

- cost performance

- low peripheral costs (e. g. for handling or clean room)

- high productivity and uptime

- ease of maintenance

These constraints have to be considered from the beginning of the system design. In what follows we will present and describe the basic sputter system design concept and show results from sputtering experiments.

\section{THE CONCEPT OF THE CIRCULUS MO}

The basic design of the CIRCULUS MO is shown in figure 1 . The system is a member of the CIRCULUS family; each of the members is dedicated to a certain disk type production, either magnetic hard disks (M) [1], phase change media (PC), or magneto optical disks (MO).

In a CIRCULUS, the vertically arranged main vacuum chamber holds either $4,8,12$ or 14 stations, which are equally distributed on a circle.

\subsection{The CIRCULUS MO}

For the CIRCULUS MO the 8 station version is used, that means 7 process stations are available for sputtering of the layer stack or for substrate etching.

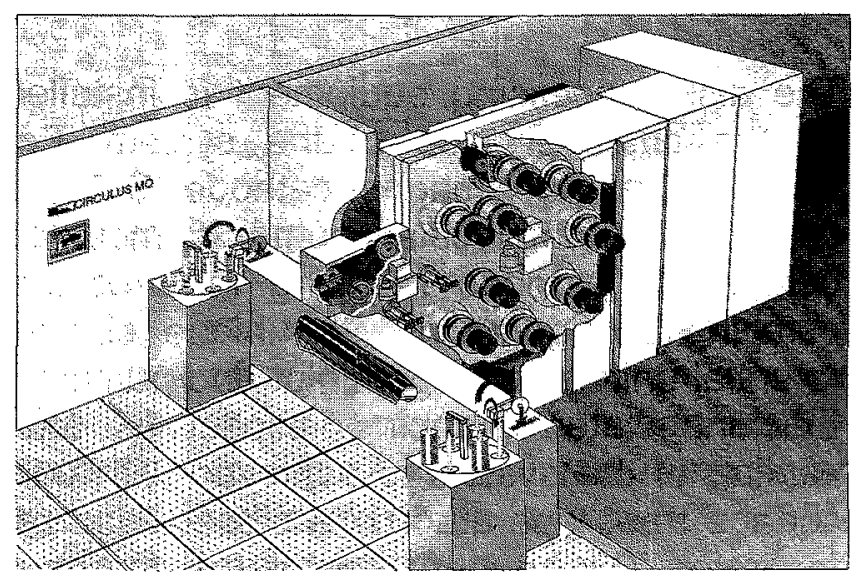

Fig. 1: Schematic drawing of the Circulus MO 
The system can be converted to different substrate sizes as $5.25^{\prime \prime}, 3.5^{\prime \prime}$ or $2.5^{\prime \prime}$ diameter. As can be seen from figure 1, the disks enter the system via a single disk loadlock and are stored in a degassing chamber, which incorporates a linear transport band. This band holds up to 360 disks, resulting in a maximum degassing time of one hour at a system cycle time of $10 \mathrm{sec}$. Leaving the degassing chamber the disks enter a small circular ring chamber (so called transfer chamber) featuring four positions. One of them is common with the $1 / 0$ of the main ring chamber. Within the transfer chamber inner and outer masks are attached to the disks (under vacuum). This integrated disk masking mechanism keeps the masks always under vacuum to avoid humidity and increased flaking by water absorption and, hence, therefore maximizes their lifetime. The masks themselves are automatically exchanged on an operator selected time basis. This is done by a mask exchange system, meaning that at one port of the transfer chamber the used masks circulating through the sputter stations can be automatically exchanged against new ones, sitting under vacuum in a mask magazine.

The main ring chamber has a central rotary drive system with 8 arms (paddles) each holding a masked disk. During process the individual process stations are - with respect to vacuum - hard-isolated from each other preventing gas cross contamination. During disk transport bypassing of the reactive gas avoids cross talk.

\subsection{Sputter station}

The disk processing equipment is installed on the chamber side of the system. For sputtering a rotary magnet cathode (CMR) is used. A turbomolecular pump is mounted behind the cathode resulting in a coaxial pumping configuration with unique circumferential uniformity of gas inlet and pumping. Efficient water pumping especially after venting of the system is achieved with cold surface traps operated by a closed loop refrigerator. The sputter target is bonded on a water-cooled backing plate. The magnetron consists of an array of permanent magnets rotating with $5 \mathrm{~Hz}$ behind the target. The gas inlet is from the disk side either through the center hole of the disk or around the disk circumference, which is expected to result in a homogenous stoichiometry over the substrate recording area [2].

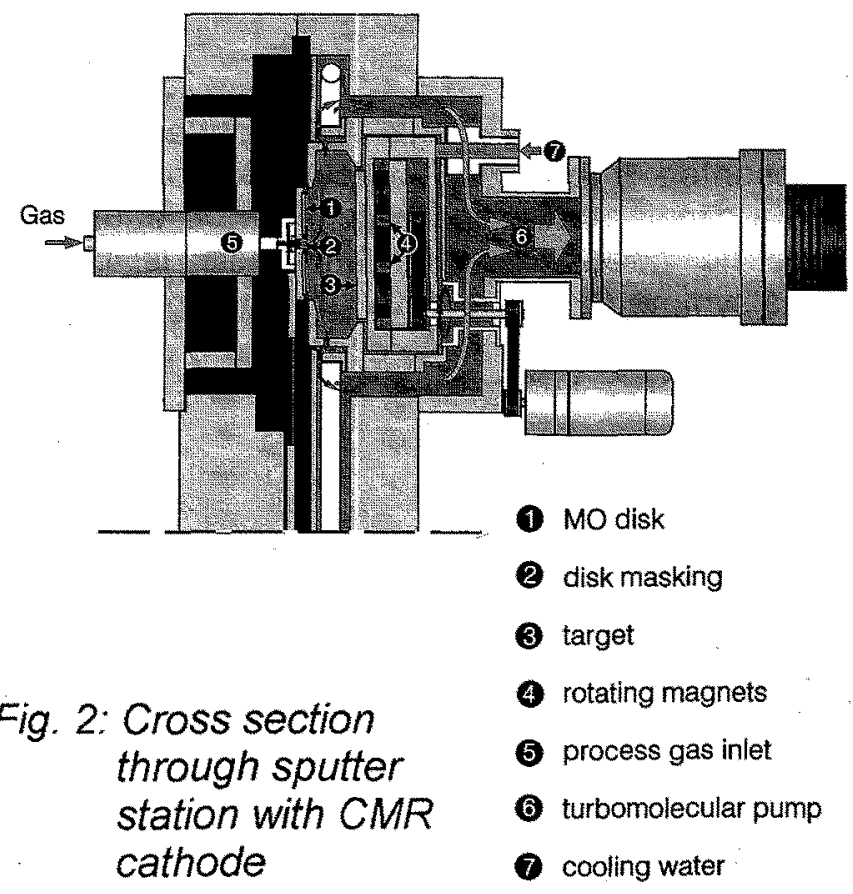

\section{REACTIVE SPUTTERING OF DIELEC- TRICS}

On magneto-optical disks the active layer is sandwiched between dielectric layers, which serve for protection, antireflection, or heat transfer. The layer stack consists, for example, of $\mathrm{Si}_{3} \mathrm{~N}_{4}-\mathrm{TbFeCo}-\mathrm{Si}_{3} \mathrm{~N}_{4}-\mathrm{Al}$ on a polycarbonate (PC) substrate. The typical requirements for the dielectric layers are:

$\begin{array}{lr}\text { layer thickness: } & \text { up to } 120 \mathrm{~nm} \\ \text { thickness uniformity: } & \pm 2.5 \% \\ \text { refractive index uniformity: } & \pm 2.0 \%\end{array}$

For the deposition of the dielectric layers the DC-reactive sputtering employing an elemental target and an argon/reactive gas mixture has proven to fulfill the requirements [3]:

- high rates $(2-5 \mathrm{~nm} / \mathrm{s})$

- low substrate (PC-disks!) temperatures

- process stability

- uniformity.

Sputtering of dielectrics means deposition of insulating layers which can undergo surface charging leading to the formation of an arc. This problem holds especially for the target 
surface and therefore the design of the CMR cathode is done in a way that the whole target surface is eroded preventing redeposition and build-up of insulating layers. Otherwise an electrical breakthrough at the dielectric layers would result in target arcing and particle emission.

A special feature of $D C$ reactive sputtering is the fact that the actual status of the cathode depends on its history, which is often called the hysteresis effect [4 - 6]. The size of this effect depends on the target material ( $\mathrm{Si}, \mathrm{Ti}$, $\mathrm{Al}, \mathrm{Ta})$, the reactive gas $\left(\mathrm{O}_{2}\right.$ or $\left.\mathrm{N}_{2}\right)$ and the design of the reactor (e. g. pumping speed).

\subsection{Sputtering of $\mathrm{Si}_{3} \mathrm{~N}_{4}$}

From the viewpoint of $\mathrm{DC}$ reactive sputtering the deposition of $\mathrm{Si}_{3} \mathrm{~N}_{4}$ from a Si-target with nitrogen as reactive gas is relatively easy due to the low reactivity.

The voltage - nitrogen flow relation in fig. 3 shows a smooth voltage decrease with increasing gas flow. Therefore the cathode can be operated in a stable mode over a wide range of $\mathrm{N}_{2}$ flows. Due to the higher secondary electron emission coefficient the discharge voltage is lower for the nitrided surface.

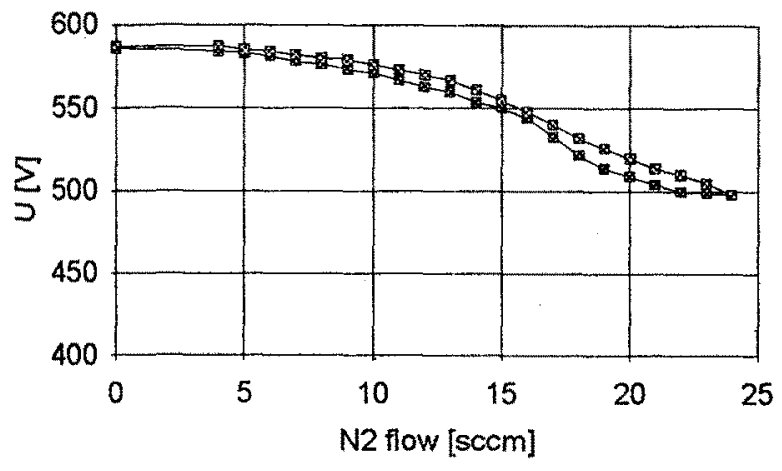

Fig. 3: Discharge voltage versus nitrogen flow for the Si-target

The lower reactivity of nitrogen to the Si-target surface results in the non-appearance of the hysteresis effect. Therefore the requirements for $\mathrm{Si}_{3} \mathrm{~N}_{4}$ deposition are weak and layer stoichiometries with different refractive indices ( $n=1.95 \ldots 2.15$ ) can be easily prepared by selecting nitrogen flow and power appropriately.

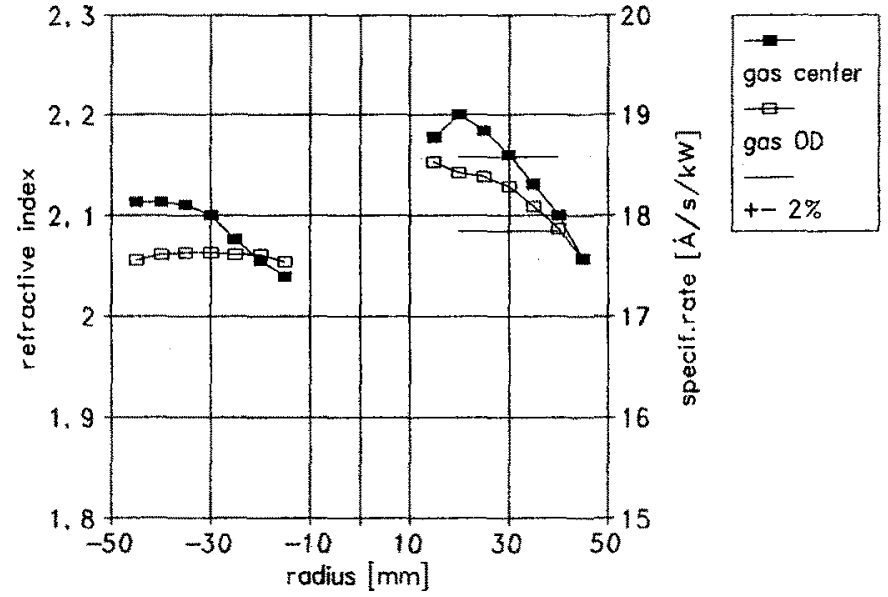

Fig. 4: Comparison of refractive index and thickness uniformity for gas inlet through the center hole (gas center) or around the circumference (gas $O D$ ) of the $3.5^{\prime \prime}$ disk

A special feature of the CIRCULUS MO is the position of the gas inlet, which is on the disk side and two inlet modes have been investigated: one through the center hole of the disk and the other arround the disk. Both are compared in figure 3 . In the first case a negative gradient of the refractive index was observed, whereas the second version gives satisfying results with uniformities of $2.0 \%$ for the thickness and of better than $1 \%$ for the refractive index. The second version is now the standard and figure 5 shows the achieved radial and circumferential uniformities. The typical deposition rates are $3-4 \mathrm{~nm} / \mathrm{s}$.

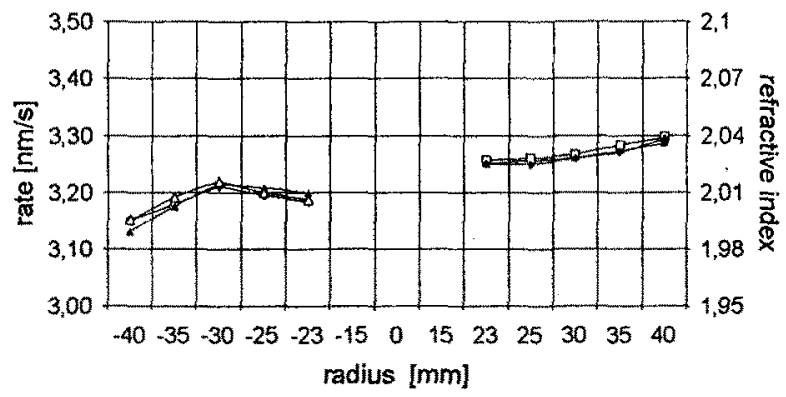

Fig. 5: Typical rate and refractive index uniformity of $\mathrm{Si}_{3} \mathrm{~N}_{4}$ deposited on an $86 \mathrm{~mm}$ MO disk by means of a sputter station according to Fig. 2. The measurements at one radius refer to three angles $10^{\circ}, 120^{\circ}$, $240^{\circ}$ ) on the circumference. 


\subsection{SPUTTERING OF OTHER DIELEC- TRICS}

Deposition of other dielectrics e. g. $\mathrm{Ta}_{2} \mathrm{O}_{5}$ is more complicated due to the higher reactivity of the involved elements. The hysteresis effect is demonstrated in figure 6 using the sputter station arrangement shown in fig. 2 with a Ta-target and oxygen for the reactive deposition of $\mathrm{Ta}_{2} \mathrm{O}_{5}$. Shown is the discharge voltage versus the oxygen flow at a constant power of $2.0 \mathrm{~kW}$. Starting with the metallic target the voltage increases with a relative smooth transition into the oxidic mode. Ta behaves like $\mathrm{Ti}$ : the secondary electron emission coefficient is higher for the metallic than for the oxidic target. Therefore, the voltage is higher in oxidic mode. Reduction of the oxygen flow gives the hysteresis effect with a sharp transition back into the metallic mode.

The second curve relates to the same set-up of the cathode, but now the vacuum pumping. capacity has been increased by a factor of three. The hysteresis gets very small and also the transition from oxidic to metallic mode is smooth, too, which allows a stable operation of the cathode. As a result of the increased pumping speed the loop is shifted to higher oxygen flows, too.

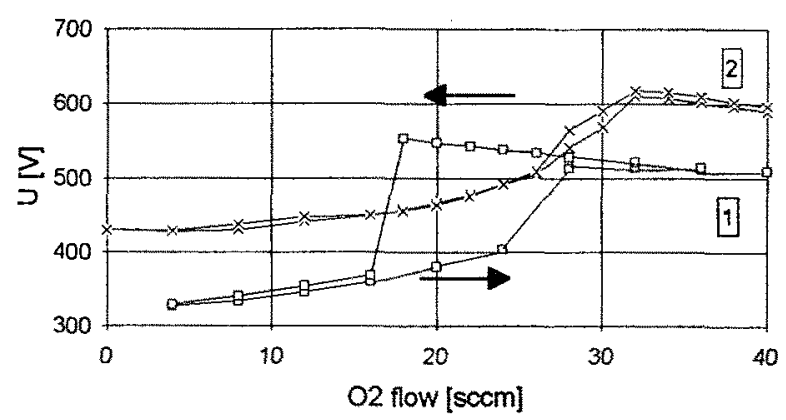

Fig. 6: Voltage versus oxygen flow relation for a Ta-target, showing the hysteresis effect. In curve 2 the vacuum pumping speed has been increased by a factor of three.

A high rate and a stoichiometric layer is obtained at the transition point from the oxidic to the metallic mode. Deposition rates of 7 $\mathrm{nm} / \mathrm{s}$ have been achieved under stable process conditions consistently over time and without arcing. Additionally, the process was pulsed (power supply on/off, as is required for a single-disk process) with no negative effect on stability.

The influence of oxygen flow on deposition rate and refractive index of the deposited $\mathrm{Ta}_{2} \mathrm{O}_{5}$ is shown in Fig. 7 for two different power levels.

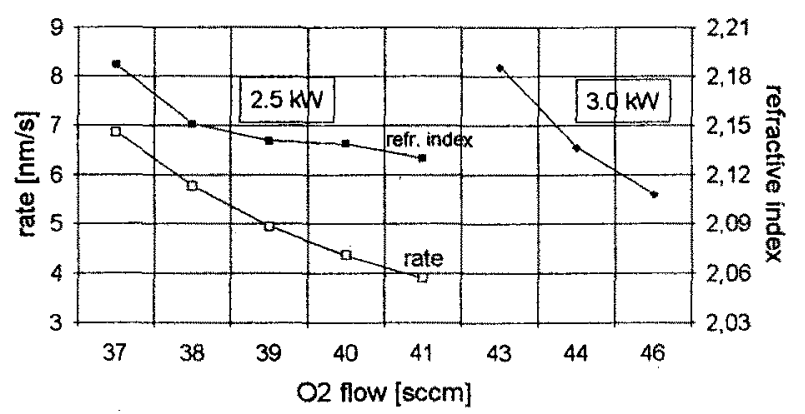

Fig 7: Dependence of deposition rate and refractive index on the oxygen flow for two different sputter powers

A careful control of the oxygen flow is required especially for the stabilization of the rate. This is achieved after conditioning of the target and reaching of thermal equilibrium of its environment. Figure 8 shows the layer uniformities achieved at a depostion rate of $8.6 \mathrm{~nm} / \mathrm{sec}(\mathrm{N}=2.5 \mathrm{~kW})$ and demonstrates, in combination with the dependencies in fig. 7 , the high degree of circumferential uniform gas supply.

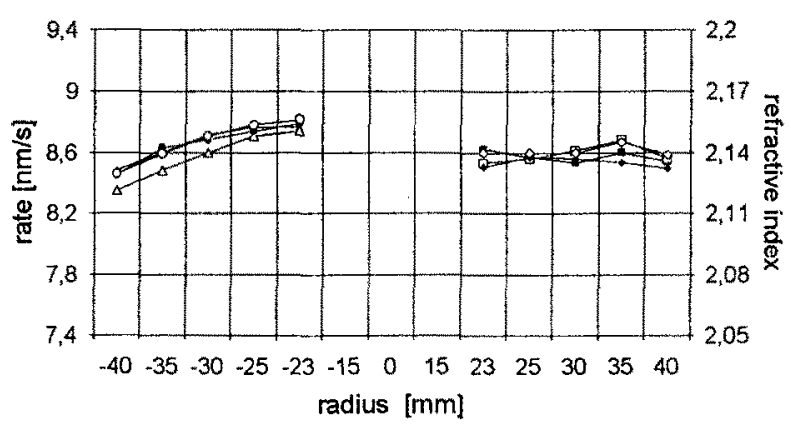

Fig. 8: Radial and circumferential thickness and refractive index uniformity for a $\mathrm{Ta}_{2} \mathrm{O}_{5}$ layer deposited on the recording area of an $86 \mathrm{~mm}$ diameter magneto-optical disk. The measurements at one radius refer to three angles $\left(0^{\circ}, 120^{\circ}, 240^{\circ}\right)$ on the circumference. 


\subsection{Process stabilities}

The time dependence of the layer deposition process is related to the stability of the components involved such as power supplies, gas flowmeters, target erosion and also the temperature of the sputter environment. Due to the stringent requirements for MO-disk layer properties the process has to be kept extremely stable.

In figures 9 and 10 the thickness of the $\mathrm{Si}_{3} \mathrm{~N}_{4}$-or $\mathrm{MO}$-layer has been measured at 9 points on the disk during the continuous operation of the system with each 10th disk being a test disk. The achieved uniformity of $\pm 2.0 \%$ on the disk can also be maintained over time which guarentees stable production conditions.

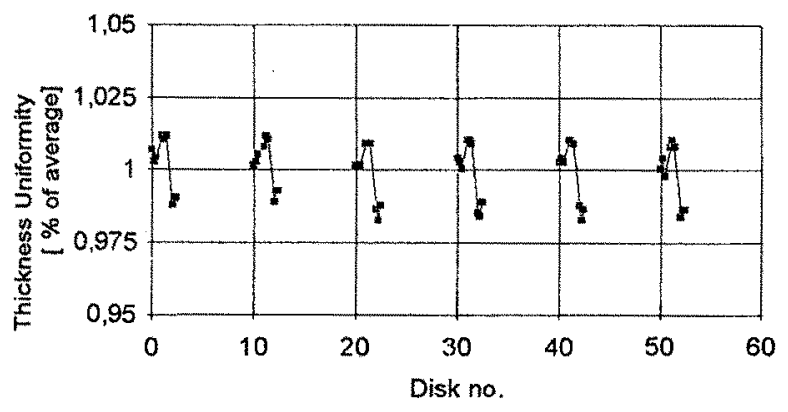

Fig. 9: Thickness uniformity of $\mathrm{Si}_{3} \mathrm{~N}_{4}$ (measured at 9 points on the disk, 3 along radius under 3 different angles) on test disks during continuous operation of the sputter system

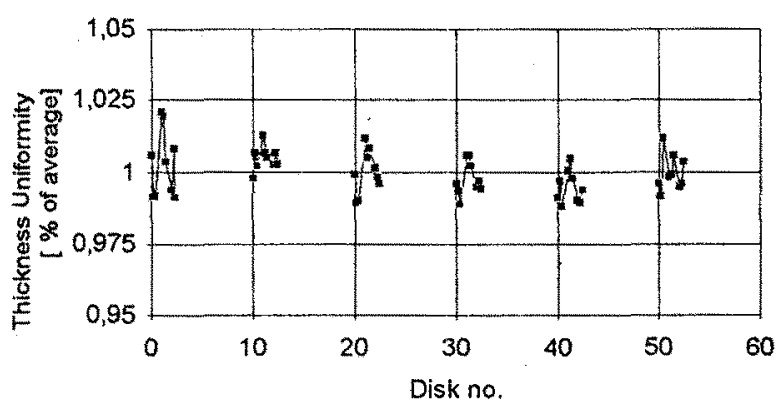

Fig 10: Same as fig. 9 but for the MO layer

\section{CONCLUSION}

A new single disk system, the CIRCULUS $\mathrm{MO}$, for the coating of magneto-optical media was presented. At the design of the system emphasis was put on fully automatic operation. Individual disks are loaded to the system, which performs internally the disk masking. The sputter process - DC-reactive for dielectrics and DC magnetron for the other layers - has been integrated and very good layer uniformities have been achieved.

\section{References}

[1] S. Schulz, B. Cord, and H. Zahel, Proceedings of the 35th Technical Conference of the Society of Vacuum Coaters (1992), Baltimore, USA

[2] C.D. Tsiogas and J.N. Avaritsiotas J. Appl.Phys. 71(10), 1992, p5173

[3] G. Bräuer, W. Dicken, and S. Müller SPIE, vol. 1323, Optical Thin Films III (1990), p. 151

[4] S. Berg, H.-O. Blom, M. Moradi, and C. Nender, J. Vac. Sci. Technol. A 7 (3), 1989, p. 1225

[5] M. Bhushan, J. Vac. Sci. Technol. A 5 (5), 1987, p. 2829

[6] S. Kadlec, J. Musil, and J. Vyskocil Vacuum, vol. 37 (1987) 10, p. 729 